\title{
Efficacy of Herbal Extracts against Enterococcus faecalis on a Dentinal Biofilm
}

\author{
Nikita Kangabam ${ }^{1}$, Arunajatesan Subbiya ${ }^{2}$, Nagarajan Geethapriya ${ }^{3}$, Kesavaram Padmavathy ${ }^{4}$, Krishnan Mahalakshmi ${ }^{5}$, \\ Ravindranath Megha ${ }^{6}$
}

\begin{abstract}
Aim and objectives: Antibacterial activity of five different extracts of Cinnamomum zeylanicum was estimated and compared with the conventional root canal irrigant sodium hypochlorite on planktonic cells as well as on a 6-week biofilm.

Materials and methods: An estimated $10 \mathrm{~g}$ of the five different herbal extracts, namely methanol, ethanol, isopropyl alcohol, acetone, and chloroform were weighed and mixed with $20 \mathrm{~mL}$ of inert solvent. Zone of inhibition was assessed by the agar well diffusion assay and MIC (minimum inhibitory concentration) by the broth dilution assay. A total of 90 teeth samples were inoculated with E. faecalis (ATCC 29212) and were incubated for 6 weeks. The samples were assigned into six different groups, namely Cinnamomum zeylanicumm ethanolic extract, Cinnamomum zeylanicum ethanolic extract, Cinnamomum zeylanicum isopropyl alcohol extract, Cinnamomum zeylanicum acetone extract, Cinnamomum zeylanicum chloroform extract, and $\mathrm{NaOCl}$. Time required to inhibit 6-week biofilm of E. faecalis was assessed using time kill curve with a time interval of 5, 10, 15, and 20 minutes. Statistical analysis was done using the Kruskal-Wallis test.

Results: The methanol, ethanol, isopropyl alcohol, acetone, and chloroform showed a complete eradication of Enterococcus faecalis-both planktonic organism and the 6-week biofilm.

Conclusion: According to the study conducted, methanol, ethanol, isopropyl alcohol, acetone, and chloroform extracts of Cinnamomum zeylanicum were found to be effective antibacterial agents against $E$. faecalis - both planktonic cells and 6 weeks biofilm formed on dentin substrate.

Clinical significance: Since cinnamon extract has a good antimicrobial activity almost at par with $\mathrm{NaOCl}$, it could be a promising alternative to conventional irrigants.
\end{abstract}

Keywords: Antimicrobial effect, Cinnamon, Dentinal biofilm, E. faecalis, Herbal extracts, Laboratory research.

Journal of Operative Dentistry and Endodontics (2019): 10.5005/jp-journals-10047-0070

\section{INTRODUCTION}

Effective debridement and disinfection of the root canal system is the key to achieve a predictable long-term success of endodontic treatment. ${ }^{1}$ Enterococcus faecalis is the most commonly cultured organism from failed endodontic treatment, and is the dominant species associated with secondary infections. ${ }^{2-5}$ It can also survive by genetic polymorphism and by its ability to bind to dentin, invade dentinal tubules, and survive starvation. ${ }^{2}$ The collaboration of shaping with instruments and cleaning with irrigants facilitates root canal disinfection. ${ }^{6,7}$ The most effective method to eliminate E. faecalis is the use of $3 \%$ to full-strength sodium hypochlorite and $2 \%$ chlorhexidine as irrigating solutions. ${ }^{2,6,7}$ But both these irrigants possess some undesirable properties such as toxicity to the periradicular tissues, reduction in dentin strength due to its proteolytic effect, allergic potential, unpredictable efficacy during storage, and unpleasant taste and odour. ${ }^{8}$ This demands the need for a search of endodontic irrigant with a better biocompatibility and antibacterial property to overcome the limitations of the currently used chemical irrigants. Herbal products are gaining popularity in every field of medicine, mainly due to their biocompatibility. They also possess high medicinal properties such as antioxidant, antimicrobial, and anti-inflammatory properties. ${ }^{9-13}$ The cinnamon tree (including the bark, leaves, flowers, fruits, and roots) has also been reported to possess antimicrobial property and biocompatibility. ${ }^{10,14,15}$ Therefore, the aim of this in vitro study is to evaluate the efficacy of different extracts of Cinnamomum zeylanicum (CZ), namely methanol, ethanol, isopropyl alcohol (IPA),
${ }^{1}$ Private Practice, Manipur, India

2,3,6 Department of Conservative Dentistry and Endodontics, Sree Balaji Dental College and Hospital, Bharath Institute of Higher Education and Research, Chennai, Tamil Nadu, India

4,5 Department of Microbiology, Research Laboratory for Oral and Systemic Health, Sree Balaji Dental College and Hospital, Bharath Institute of Higher Education and Research, Chennai, Tamil Nadu, India Corresponding Author: Arunajatesan Subbiya, Department of Conservative Dentistry and Endodontics, Sree Balaji Dental College and Hospital, Bharath Institute of Higher Education and Research, Chennai, Tamil Nadu, India, Phone: +91 9840197437, e-mail: drsubbiya@gmail. com

How to cite this article: Kangabam N, Subbiya A, et al. Efficacy of Herbal Extracts against Enterococcus faecalis on a Dentinal Biofilm. J Oper Dent Endod 2019;4(1):22-26.

Source of support: Nil

Conflict of interest: None

acetone, and chloroform extracts against a 6-week Enterococcus faecalis dentinal biofilm.

\section{Materials and Methods}

\section{Bacterial Strain Used}

The stock culture of E. faecalis (ATCC 29212) that had been maintained at the Department of Microbiology, Sree Balaji Dental 
College and Hospital, Chennai, India was used for the study. The cultures were revived on Muller Hinton Agar (MHA) and checked for purity.

\section{Preparation of Herbal Extracts}

Extracts of $C$. zeylanicum were prepared $(2: 1 \mathrm{w} / \mathrm{v})$ by the cold percolation method using the following solvents: ethanol, methanol, acetone, chloroform, and isopropyl alcohol. The herbal extracts were evaporated to dryness and the stock solution of the extracts were prepared using 10\% dimethyl sulfoxide (DMSO). The stock solutions were filter sterilized using Sartorius Minisart ${ }^{\circledR}$ syringe filters.

\section{Screening for Antibacterial Activity}

The antibacterial activity of the herbal extracts was assessed by well diffusion assay. An inoculum was prepared by the colony suspension method as per CLSI guidelines. ${ }^{16}$ The turbidity was adjusted using sterile MHB to match 0.5 McFarland standard so as to achieve a cell density of $1.5 \times 10^{8}$ cells. Lawn culture of E. faecalis was prepared on sterile MHA plates and wells of $8 \mathrm{~mm}$ diameter were punched out aseptically using a sterile cork borer. A volume of $200 \mu \mathrm{L}$ of herbal extract solutions at desired concentrations was introduced into the well. The agar plates were incubated at $37^{\circ} \mathrm{C}$ for $16-18$ hours. The zone of inhibition diameter was measured by graduated scale. Sodium hypochlorite (2.5\%) was included as positive control.

\section{Determination of MIC}

The MIC of CZ against E. faecalis (ATCC 29212) was assesssed by a broth microdilution assay. Briefly, double serial dilutions $(100,50$, $25,12.5,6.25,3.125 \mathrm{mg} / \mathrm{mL}$ ) of the herbal extracts were prepared from well 1 to well 11 of each row in sterile microtitre plates. The last well served as the culture control. The first 11 wells contained doubling solutions of herbal extracts. To all the wells, $10 \mu \mathrm{L}$ of the broth culture of E. faecalis (ATCC 29212) was added and plate was incubated at $37^{\circ} \mathrm{C}$ overnight. The MIC was regarded as the lowest concentration in the series of dilution, which did not permit the growth of $E$. faecalis.

\section{Preparation of Tooth Samples}

Forty-five freshly extracted intact, noncarious, single-rooted human mandibular premolars with fully formed apices were chosen for the study. The teeth were cleaned, radiographed, and stored in normal saline. The tooth specimens were sectioned below the cemento-enamel junction (CEJ) with a diamond disc to obtain a standard tooth length of $8 \mathrm{~mm}$. A 10-K file was used for scouting, establishing patency, and for establishing the working length. All the samples were instrumented using protaper rotary to F3. During instrumentation, $2 \mathrm{~mL}$ of $3 \% \mathrm{NaOCl}$ solution was used as a working solution delivered by a 30-gauge side-vent needle placed as far possible into the canal without binding. The samples were vertically sectioned along the mid-sagittal plane into two halves using a chisel and mallet.

\section{Sterilization of the Prepared Tooth Samples}

The prepared tooth samples were placed in clean glass test tubes containing $5 \mathrm{~mL}$ of $\mathrm{MHB}$ and autoclaved at $121^{\circ} \mathrm{C}$ at $15 \mathrm{lbs}$ pressure for 20 minutes. The efficacy of sterilization was assessed by including a biological indicator, Geobacillus stearothermophilus MTCC 1518. After sterilization, the tubes were incubated at $37^{\circ} \mathrm{C}$ for 48 hours and observed for turbidity to double check the sterility of the samples.

\section{Formation of 6-week Dentinal Biofilm}

The tooth samples in test tubes containing sterile MHB were inoculated with $10 \mu \mathrm{L}$ of $E$. faecalis ATCC 29212, cultured overnight at $37^{\circ} \mathrm{C}$ in Mueller Hinton broth adjusted to an optical density of $1.5 \times 10^{8}$ cells $/ \mathrm{mL}$ with $0.5 \mathrm{McFarland}$ standard. To avoid nutrient depletion and accumulation of toxic end products, the culture medium (MHB) was replaced every alternate day. The culture purity was checked by inoculating a loopful of culture media onto Mueller Hinton agar and using Gram staining.

At the end of 6th week, the incubated samples were randomly assigned to all the following 6 groups of test solutions and were used for quantitative assay. The test groups are categorized based on various extracts of Cinnamomum zeylanciumas: group Imethanol, group II-ethanol, group III-IPA, group IV—acetone, group $\mathrm{V}$-chloroform, group $\mathrm{VI}$-positive control (2.5\% NaOCI), group VII-negative control.

\section{Time Kill Assay}

Tooth samples $(n=20)$ with a six-week-old biofilm were grouped into 5 groups of 4 each. The planktonic cells on the tooth samples were removed by rinsing twice with sterile saline. The dentinal biofilm formed were scraped with the help of a sterile scalpel and inoculated into $1 \mathrm{~mL}$ of MHB, gently vortexed. The spread plate method was adopted to determine the colony forming units (cfu/mL) in the control (no exposure to herbal extracts).

To determine the antibiofilm activity of the herbal extracts, the tooth samples (planktonic cells removed) were immersed in the herbal extracts at a concentration of $6 \times$ MIC against the planktonic cells and $\mathrm{cfu} / \mathrm{mL}$ was determined by the spread plate method at various time intervals ( 5 minutes, 10 minutes, 15 minutes, and 20 minutes).

\section{Statistical Analysis}

Statistical analysis was conducted using a one-way ANOVA and post hoc test in SPSS 21.0 software. Descriptive data were given as mean $\pm S D$; Mann Whitney $U$ test was done for inter-group comparison. A $p$ value of $<0.05$ was considered to be statistically significant.

\section{Results}

The antibacterial activity of the different extracts of $C Z$ against E. faecalis ATCC 29212 are depicted in Figure 1. Among the different extracts of CZ tested, acetone extract exhibited higher antibacterial activity followed by IPA, ethanol, chloroform, and methanol in the decreasing order. Post hoc tests revealed four homogenous subsets. As the Mann-Whitney $U$ test revealed, statistical difference was observed in the antibacterial activity of IPA, ethanol, and chloroform extracts of CZ against E. faecalis ATCC 29212.

Of all the MICs of different extracts of CZ tested against E. faecalis ATCC 29212, the ethanol extract exhibited the least MIC $(12.5 \mathrm{mg} / \mathrm{mL})$, acetone extract showed an MIC of $25 \mathrm{mg} / \mathrm{mL}$ followed by methanol $(50 \mathrm{mg} / \mathrm{mL})$, IPA $50(\mathrm{mg} / \mathrm{mL})$, and chloroform extracts (100 mg/mL) (Table 1).

Time-dependent gradual reduction in viable bacterial count was observed in all the test groups (Fig. 2). Pairwise comparisons using Mann-Whitney $U$ test showed a statistically significant difference in the mean viable count of E. faecalis ATCC 29212 exposed to the chloroform extract of $C$. zeylanicum compared to the other extracts at both 5-minute and 10-minute exposure time (chloroform vs methanol, $p=0.05$; chloroform vs ethanol, $p=0.037$; 


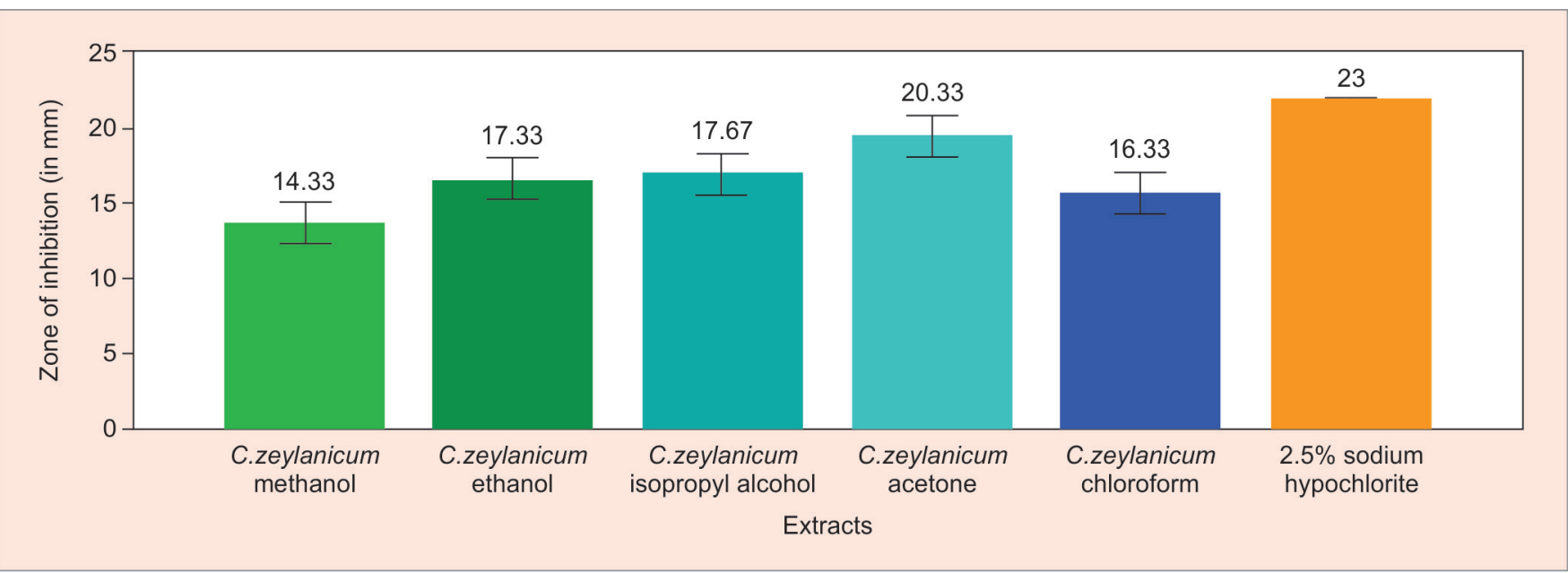

Fig. 1: Antibacterial activity of the extracts of C. zeylanicum against E. faecalis ATCC 29212

Table 1: Zone of inhibition and minimal inhibition concentration

\begin{tabular}{lll}
\hline $\begin{array}{l}\text { C. zeylanicum } \\
\text { extracts } 100 \mathrm{mg}\end{array}$ & Zone of inhibition & $\begin{array}{l}\text { Minimal inhibition } \\
\text { concentration }\end{array}$ \\
\hline Methanol & $14 \mathrm{~mm}$ & $50 \mathrm{mg}$ \\
Ethanol & $18 \mathrm{~mm}$ & $12.5 \mathrm{mg}$ \\
Isopropyl alcohol & $18 \mathrm{~mm}$ & $50 \mathrm{mg}$ \\
Acetone & $20 \mathrm{~mm}$ & $25 \mathrm{mg}$ \\
Chloroform & $16 \mathrm{~mm}$ & $100 \mathrm{mg}$ \\
$2.5 \% \mathrm{NaOCl}$ & $23 \mathrm{~mm}$ & $0.005 \%$ \\
\hline
\end{tabular}

chloroform vs isopropylalcohol, $p=0.037$; chloroform vs acetone, $p=0.037)$. Antibiofilm activity of chloroform extract of $C Z$ was almost in par with the activity of the conventional irrigant sodium hypochlorite at 10,15 , and 20 minutes $(p=1.000)$.

\section{Discussion}

A 6-week biofilm of $E$. faecalis was chosen to evaluate the antibacterial efficacy because a 6-week duration resulted in a matured biomineralized biofilm, which could make it more resistant to antimicrobial agents. ${ }^{17,18}$ Various studies have demonstrated numerous beneficial health effects of $C Z$, such as anti-inflammatory properties and antimicrobial activity..$^{10,12,13,15} \mathrm{CZ}$ was also found to have cytocompatiblity to L929 fibroblasts and has shown to have a potential as an antimicrobial agent. ${ }^{19}$

The main components of the cinnamon bark extract are cinnamaldehyde, followed by cinnamyl alcohol, terpinen-4-ol, eugenol, linalool, cinnamyl acetate, o-pinene, limonene, and $\beta$-caryophyllene. ${ }^{20-22}$ In this study, the efficiency of different solvents to prepare the extracts of $C Z$ has been investigated because different herbal extracts contain different types of active compounds such as glycosides, alkaloids, tannins, resins, saponins, coumarine, flavones, phenols, and terpens. Therefore, we have used five different types of extracts in cinnamon and evaluated their antibacterial efficacy against $E$. faecalis. The ethanolic extracts of CZ bark can be sources of trimeric, and higher oligomericproanthocyanidins, protocatechuic acid, as well as caffeic, chlorogenic, and cinnamic acids. ${ }^{21}$ Cinnamaldehyde possesses antimicrobial effects, as it can inhibit cell wall

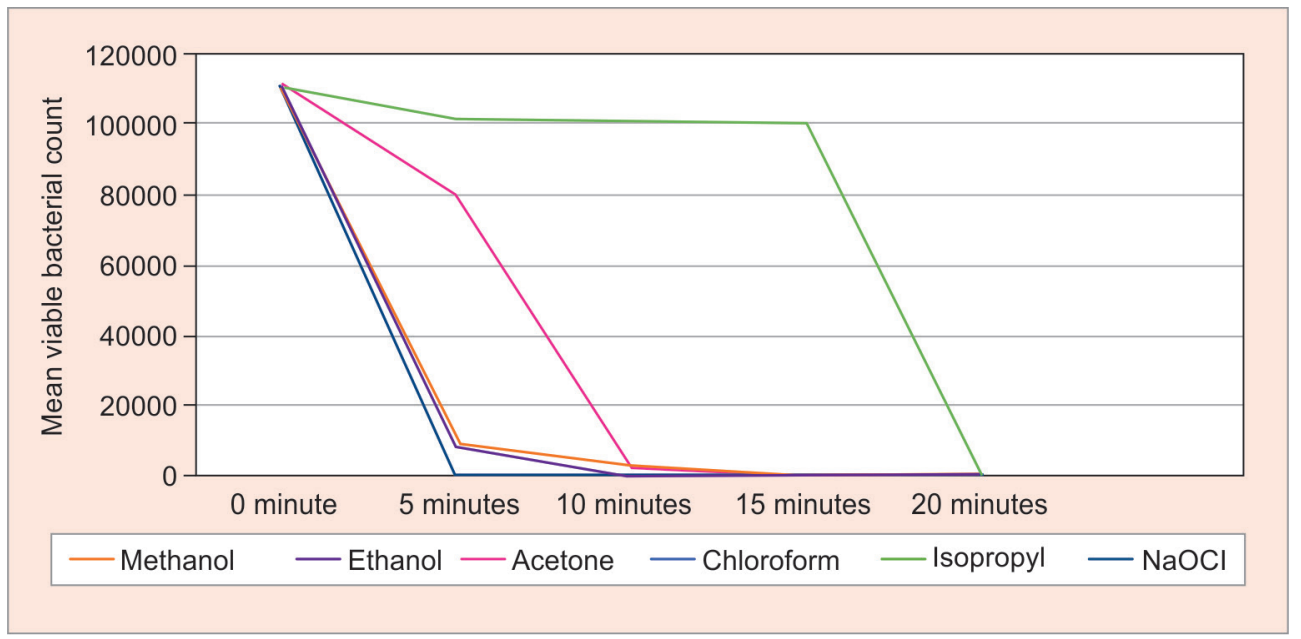

Fig. 2: Mean viable bacterial count vs time of all the extracts. Note: Chloroform almost exactly matches with NaOCl-viable bacterial count is almost the same between the two groups at all time intervals except at 5 minutes 
Table 2: Mean of viable bacterial count at different time intervals (in triplicate)

\begin{tabular}{|c|c|c|c|c|c|c|c|c|c|c|}
\hline \multirow{4}{*}{$\begin{array}{l}\text { C. zeylanicum } \\
\text { extract }\end{array}$} & \multicolumn{10}{|c|}{ Time } \\
\hline & \multicolumn{2}{|c|}{0 minute } & \multicolumn{2}{|c|}{5 minutes } & \multicolumn{2}{|c|}{10 minutes } & \multicolumn{2}{|c|}{15 minutes } & \multicolumn{2}{|c|}{20 minutes } \\
\hline & \multicolumn{2}{|c|}{ Bacterial count } & \multicolumn{2}{|c|}{ Bacterial count } & \multicolumn{2}{|c|}{ Bacterial count } & \multicolumn{2}{|c|}{ Bacterial count } & \multicolumn{2}{|c|}{ Bacterial count } \\
\hline & Mean & $S D$ & Mean & $S D$ & Mean & $S D$ & Mean & $S D$ & Mean & $S D$ \\
\hline Methanol & 110,000 & 0 & 10,000 & 200 & 3,200 & 100 & 0 & 0 & 0 & 0 \\
\hline Ethanol & 110,000 & 0 & 10,000 & 200 & 3,200 & 100 & 0 & 0 & 0 & 0 \\
\hline Acetone & 110,000 & 0 & 80,000 & 954 & 2,100 & 200 & 0 & 0 & 0 & 0 \\
\hline Chloroform & 110,000 & 0 & 100 & 0 & 0 & 0 & 0 & 0 & 0 & 0 \\
\hline Isopropyl alcohol & 110,000 & 0 & 101,000 & 2,606 & 100,100 & 2,007 & 100,000 & 2,000 & 0 & 0 \\
\hline Hypochlorite & 110,000 & 0 & 0 & 0 & 0 & 0 & 0 & 0 & 0 & 0 \\
\hline
\end{tabular}

biosynthesis, membrane function, and specific enzyme activities. Tannins are a class of compounds that are effective against C. zeylanicum; the antimicrobial activity of these compounds may be related to their ability to inactivate microbial adhesins, enzymes, and transport proteins. ${ }^{21,23}$ All the five types of solvents studied were proved to be effective against $E$. feacalis (Table 2).

In this study, for a qualitative analysis of antimicrobial efficacy, an agar well diffusion assay was used. To determine the MIC, the microdilution method was used. The efficacy of extracts was determined by the time kill assay. The time kill studies provide valuable information on rate, concentration, and potential action of antibacterial agents in vitro.

The results of the agar well diffusion assay showed that all the groups inhibited the growth of E. faecalis. In the agar well diffusion assay, the acetone extract exhibited higher antibacterial activity followed by IPA, ethanol, chloroform, and methanol in decreasing order (Table 1). A statistically significant difference was observed between groups $(p=0.000)$.

In the microdilution assay, CZ ethanol group (group II) had the lowest MIC, whereas chloroform (group V) had the highest MIC (Table 1). Though the results were not statistically significant, it could be clinically significant, as a very low concentration will have a minimal deleterious effect on the dentinal structure. The anti-biofilm activity was different from the results obtained from the agar well diffusion assay, which could be due to the different methods of assessment.

When the time kill assay was evaluated, sodium hypochlorite showed a complete elimination on 5-minutes exposure, while all extracts showed growth to a varied extent. The chloroform extracts were more effective against the $E$. faecalis biofilm than its efficiency during the agar well diffusion assay. The five types of extracts from different solvents studied proved to be effective in antibacterial activity against $E$. faecalis biofilm.

The effectiveness of chloroform extract could be due to the difference in the type of extracted active compounds compared to other extracts. The flavonoids and terpenoids exert an antibacterial effect by its ability to rupture the cellular membrane by forming complexes with proteins on the cell wall. ${ }^{24}$ The active compounds found in the alcohol extracts are alkaloids and terpens. The antibacterial action of the alkaloids may be due to their ability to intercalate with DNA, inhibition of enzymes (esterase, DNA-, RNA-polymerase), and inhibition of cell respiration. According to Cowan et al., flavanols have the ability to target surface-exposed adhesins, cell wall polypeptides, and membrane-bound enzymes in the microbial cells. ${ }^{23}$
This study shows that the agar well diffusion assay should be considered only as a preliminary test to evaluate the antibacterial activity, and not to be done alone. The results obtained in this study clearly shows that the time kill assay, which is clinically more relevant, differs from the agar well diffusion assay. This study has shown that cinnamon extracts can be an effective alternative to the existing antimicrobial irrigants, and among them, chloroform extract is more effective than other extracts. Further studies can be done to evaluate the active components in the cinnamon chloroform extract and the effect of such an irrigant on the physical and mechanical properties of the dentin and pulp tissue.

\section{Conclusion}

All the extracts used in this study were found to be effective antibacterial agents against $E$. faecalis planktonic cells and the 6-week biofilm formed on the dentin substrate. Among the extracts, chloroform extract is more effective and is at par with $\mathrm{NaOCl}$. This study paves the way for further attention and research to identify the active compounds responsible for the plant biological activity. Further studies should be done to explain in detail the exact mechanism of action by which extracts exert their antimicrobial effect.

\section{Clinical Significance}

Since cinnamon extract has a good antimicrobial activity almost at par with $\mathrm{NaOCl}$, it could be a promising alternative to conventional irrigants.

\section{References}

1. Sjögren U, Figdor D, et al. Influence of infection at the time of root filling on the outcome of endodontic treatment of teeth with apical periodontitis. Int Endod J 1997 Sep;30(5):297-306. DOI: 10.1111/j.13652591.1997.tb00714.x.

2. Stuart $\mathrm{CH}$, Schwartz SA, et al. Enterococcus faecalis: its role in root canal treatment failure and current concepts in retreatment. J Endod 2006 Feb 1;32(2):93-98. DOI: 10.1016/j.joen.2005.10.049.

3. Rôças IN, Siqueira Jr JF, et al. Association of Enterococcus faecalis with different forms of periradicular diseases. J Endod 2004 May 1;30(5):315-320. DOI: 10.1097/00004770-200405000-00004.

4. Siqueira Jr JF, Rôças IN. Polymerase chain reaction-based analysis of microorganisms associated with failed endodontic treatment. Oral Surg Oral Med Oral Pathol Oral Radiol Endod 2004 Jan 1;97(1):85-94. DOI: 10.1016/S1079-2104(03)00353-6.

5. Peciuliene V, Balciuniene I, et al. Isolation of Enterococcus faecalis in previously root-filled canals in a Lithuanian population. J Endod 2000 Oct 1;26(10):593-595. DOI: 10.1097/00004770-20001000000004. 
6. Kennedy J, Hussey D. The antimicrobial effects of root canal irrigation and medication. Oral Surg Oral Med Oral Pathol Oral Radiol Endod 2007 Apr 1;103(4):560-569. DOI: 10.1016/j.tripleo.2006.10.004.

7. Davis JM, Maki J, et al. An in vitro comparison of the antimicrobial effects of various endodontic medicaments on Enterococcus faecalis. J Endod 2007 May 1;33(5):567-569. DOI: 10.1016/j.joen.2007.01.015.

8. Yesilsoy $C$, Whitaker E, et al. Antimicrobial and toxic effects of established and potential root canal irrigants. J Endod $1995 \mathrm{Oct}$ 1;21(10):513-515. DOI: 10.1016/S0099-2399(06)80524-8.

9. Prabhakar J, Senthilkumar M, et al. Evaluation of antimicrobial efficacy of herbal alternatives (Triphala and green tea polyphenols), MTAD, and 5\% sodium hypochlorite against Enterococcus faecalis biofilm formed on tooth substrate: an in vitro study. J Endod 2010 Jan 1;36(1):83-86. DOI: 10.1016/j.joen.2009.09.040.

10. Nabavi S, Di Lorenzo A, et al. Antibacterial effects of cinnamon: From farm to food, cosmetic and pharmaceutical industries. Nutrients 2015;7(9):7729-7748. DOI: 10.3390/nu7095359.

11. Simoes M, Bennett RN, et al. Understanding antimicrobial activities of phytochemicals against multidrug resistant bacteria and biofilms. Nat Prod Rep 2009;26(6):746-757. DOI: 10.1039/b821648g.

12. Daglia M. Polyphenols as antimicrobial agents. Curr Opin Biotechnol 2012 Apr 1;23(2):174-181. DOI: 10.1016/j.copbio.2011.08.007.

13. Nascimento GG, Locatelli J, et al. Antibacterial activity of plant extracts and phytochemicals on antibiotic-resistant bacteria. Braz J Microbio 2000 Oct;31(4):247-256. DOI: 10.1590/S1517-83822000000400003.

14. LeBel G, Haas B, et al. Effect of cinnamon (Cinnamomum verum) bark essential oil on the halitosis-associated bacterium Solobacterium moorei and in vitro cytotoxicity. Arch Oral Biol 2017 Nov;83:97-104. DOI: 10.1016/j.archoralbio.2017.07.005.

15. Shreaz S, Wani WA, et al. Cinnamaldehyde and its derivatives, a novel class of antifungal agents. Fitoterapia 2016 Jul 1;112:116-131. DOI: 10.1016/j.fitote.2016.05.016.
16. CLSI. Performance standards for antimicrobial susceptibility testing: Twenty Eighth International Supplement, CLSI supplement M100. Wayne PA: Clinical and Laboratory Standards Institute; 2018.

17. Tong Z, Zhou L, et al. In vitro evaluation of the antibacterial activities of MTAD in combination with nisin against Enterococcus faecalis. Journal of endodontics 2011 Aug 1;37(8):1116-1120. DOI: 10.1016/ j.joen.2011.03.020

18. Kennedy J, Hussey D. The antimicrobial effects of root canal irrigation and medication. Oral Surg Oral Med Oral Pathol Oral Radiol Endod 2007 Apr 1;103(4):560-569. DOI: 10.1016/j.tripleo.2006.10.004.

19. Abbaszadegan A, Dadolahi S, et al. Antimicrobial and cytotoxic activity of cinnamomumzeylanicum, calcium hydroxide, and triple antibiotic paste as root canal dressing materials. J Contemp Dent Pract 2016 Feb;17(2):105-113. DOI: 10.5005/jp-journals-10024-1811.

20. Archer AW. Determination of cinnamaldehyde, coumarin and cinnamyl alcohol in cinnamon and cassia by high-performance liquid chromatography. J Chromatogr A 1988 Jan 1;447:272-276. DOI: 10.1016/S0021-9673(01)91485-2.

21. Jayaprakasha GK, Rao LJ. Chemistry, biogenesis, and biological activities of cinnamomumzeylanicum. Crit Rev Food Sci Nutr 2011 Jul 1;51(6):547-562. DOI: 10.1080/10408391003699550.

22. Mallavarapu G, Rao B. Chemical constituents and uses of CinnamomumzeylanicumBlume. Aromatic Plants From Asia Their Chemistry and Application in Foof and Therapy. Dehradun, India: Har. Krishan Bhalla \& Sons; 2007.

23. Cowan MM. Plant products as antimicrobial agents. Clin Microbiol Rev 1999 Oct 1;12(4):564-582. DOI: 10.1128/CMR.12.4.564.

24. Gill AO, Holley RA. Mechanisms of bactericidal action of cinnamaldehyde against Listeria monocytogenes and of eugenol against L. monocytogenes and Lactobacillus sakei. Appl Environ Microbiol 2004 Oct 1;70(10):5750-5755. DOI: 10.1128/AEM.70.10.57505755.2004 